\title{
Modelo predictor de las motivaciones para correr: análisis con corredores de ruta españoles vs. mexicanos*
}

\author{
Predictor Model for Running Motivations: Analysis \\ of Spanish vs. Mexican Route Runners
}

Recibido: enero 28 de 2014 | Revisado: febrero 5 de 2015 | Aceptado: febrero 5 de 2015

\author{
ANTONIO ZARAUZ SANCHO ** \\ I.E.S. Azcona de Almería, España \\ Francisco RUIZ-JUAN *** \\ Universidad de Murcia, España \\ FÉLIX ARBINAGA IBARZÁBAL **** \\ Universidad de Huelva, España \\ José CARLOS JAENES SÁNCHEZ ****** \\ Universidad Pablo de Olavide, España \\ GABRIEL FLORES-ALLENDE ****** \\ Universidad de Guadalajara, México
}

doi:10.11144/Javeriana.upsy14-2.mpmc

Para citar este artículo: Zarauz, A., Ruiz -Juan, F., Arbinaga, F., Jaenes, J. C., \& Flores-Allende, G. (2015). Modelo predictor de las motivaciones para correr: análisis con corredores de ruta españoles vs. mexicanos. Universitas Psychologica, 14(2), 659. 674. http://dx.doi.org.10.11144/Javeriana.upsy14-2. mpmc

\footnotetext{
* Artículo de investigación.

**_Correo electrónico: tonizarauz@msn.com

**** Correo electrónico: fruizj@um.es felix.arbinaga@dpsi.uhu.es

****** Correo electrónico: felix.arbinaga@dpsi.uhu.es

******* Correo electrónico: jcjaesan@upo.es

********* Correo electrónico: cfdgesa75@hotmail.com
}

\section{RESUMEN}

En la práctica de ejercicio físico, estudiar las motivaciones implicadas en que un sujeto desarrolle tal actividad, se ha convertido en uno de los temas fundamentales de la psicología del deporte y el ejercicio. Se pretendía conocer qué relaciones predictivas presentaban el compromiso a correr, la ansiedad competitiva y la percepción de éxito en las carreras en ruta sobre los motivos para realizarlas. Participaron 1.795 corredores de fondo en ruta (1.105 españoles, 690 mexicanos). De ellos el $85.65 \%$ fueron hombres con una edad de $M=38.98$ (DT $=10.45)$, y $14.35 \%$ mujeres, con una edad $M=37.88$ $(D T=9.80)$. La recogida de datos se llevó a cabo mediante las adaptaciones al castellano de las Motivations of Marathoners Scales-34 (MOMS-34), la Commitment to Running Scale-11 (CR-11), el Revised Competitive State Anxiety Inventory-2 (CSAI-2R) y el Perception of Success Questionnaire (POSQ). Se ha obtenido que los mexicanos, frente al grupo de españoles, alcanzaron mayores puntuaciones en todas las subescalas de las MOMS-34, en la CR11, en el POSQ y en las subescalas Autoconfianza del CSAI-2R, pero menor puntuación en ansiedad somática, no habiendo diferencias significativas en ansiedad cognitiva. Por último, los siete modelos predictivos basados en los análisis de regresión fueron muy similares cuando se compararon las dos nacionalidades agrupadas por el sexo del participante, obteniéndose unas varianzas explicativas muy altas. Pero se mostraron como modelos predictivos diferenciales cuando se compararon los hombres vs. las mujeres.

Palabras clave

ansiedad; autoconfianza; compromiso; ego; tarea

\section{A B S T R ACT}

In physical exercise, studying the incentives involved when a subject is developing within their activity has become one of the key concerns of sport and exercise psychology. The aim was to establish the predictive effects of commitment to running, competitive anxiety and perception of success in route races upon the reasons for participating in them. 1.795 long-distance runners took part (1.105 Spanish, 690 Mexicans). Of these, $85.65 \%$ were men with an average age of $M=38.98(D T=10.45)$, and $14.35 \%$ were wo- 
men, with an average age of $M=37.88(D T=9.80)$. Data were collected via Spanish language adaptations of Motivations of Marathoners Scales-34 (MOMS-34), the Commitment to Running Scale-11 (CR-11) the Revised Competitive State Anxiety Inventory-2 (CSAI-2R) and the Perception of Success Questionnaire (POSQ). The results showed that compared to the Spanish group, the Mexicans scored higher in all sub-scales of the MOMS-34, the CR-11, the POSQ and in the Self-confidence sub-scales of the CSAI-2R, but lower in Physical anxiety. There were no significant differences in terms of Cognitive anxiety. Finally, the seven predictive models based on regression analyses were very similar when the two nationalities were compared in groups according to the participants' sex, producing some very high explanatory variance. But the predictive models were shown to be differential when men were compared with women.

Keywords

anxiety; self-confidence; commitment; ego; task

\section{Introducción}

En la práctica de ejercicio físico, y en su caso en el deporte, estudiar los aspectos motivacionales implicados en que un sujeto desarrolle una actividad, se ha convertido en uno de los temas fundamentales de la psicología del deporte y el ejercicio. Ya Gould (1980) sugería la necesidad de llevar a cabo estudios que permitan identificar la variedad de motivos y sus relaciones al involucrarse en equipos deportivos. Ahora bien, hay que ser conscientes de que estos motivos de inicio (Isorna, Rial, Vaquero-Cristóbal, \& Sanmartín, 2012; López \& Márquez, 2001) no tienen por qué coincidir con aquellos alegados para mantenerse (Castillo, Balaguer, \& Duda, 2000; Isorna, Rial \& Vaquero-Cristóbal, 2014; MartínAlbo, Núñez, \& Navarro, 2003), para abandonar (Allied Dunbar National Fitness Survey, 1992; Zamarripa, Ruiz-Juan, López, \& Fernández, 2013) o para el cambio de un deporte por otro (MartínAlbo \& Núñez, 1999). Así, se alega que aquellos estarían sometidos a una evolución, en función del tiempo de práctica, interactuando tanto aspectos personales como situacionales (Escartí \& Brustad, 2000). Desde esta perspectiva, un elemento diferenciador de los deportes podría ser la evolución de los motivos a lo largo de su práctica; lo cual permitiría llevar a cabo clasificaciones de los mismos (MartínAlbo, Núñez, \& Navarro, 1997).
Con anterioridad Vanek y Cratty (1970) habían hecho intentos por organizar los diversos motivos alegados desde una perspectiva evolutiva a lo largo de la carrera deportiva, diferenciando cuatro fases: generalización, diferenciación, especialización e involución. Posteriormente Martín-Albo y Núñez (1999) propusieron un modelo evolutivo también de cuatro etapas: inicio, mantenimiento, cambio y abandono. Fue en este contexto donde surgió un amplio número de clasificaciones de motivos, especialmente para la iniciación en la práctica deportiva (Castillo et al., 2000; Isorna et al., 2014).

Crandall (1980) había realizado una llamada de atención sobre la necesidad de estudiar específicamente los motivos de práctica en los diferentes deportes. Analizando las relaciones entre los antecedentes motivacionales y problemas derivados de la práctica de ejercicio en corredores, Fisher (2001) y Ogden, Veale y Summers (1997) sugirieron que las características motivacionales y de dependencia que presentaban los deportistas en ejercicios aeróbicos no podían generalizarse a la población que lleva a cabo entrenamientos de fuerza. Se llegó a considerar que los diferentes tipos de ejercicios tienen diferentes objetivos y factores motivacionales (Crandall, 1980; González-Badillo \& Ribas, 2002; González-Badillo \& Goristiagoa, 2002).

Por ello, al estudiar los aspectos motivacionales es conveniente que el interés se centre de manera específica en el deporte o ejercicio que se practica. En este sentido, Masters, Ogles y Jolton (1993) desarrollaron, para pruebas de maratón, el instrumento Motivations of Marathoners Scales (MOMS) que evalúa cuatro categorías generales -motivos psicológicos, de logro, sociales y de salud física- y nueve categorías más específicas de motivos -salud general, concerniente al peso, afiliación, reconocimiento, competición, logro de metas personales, metas psicológicas, autoestima y sentido de la vida-. En el caso de las carreras de larga distancia, utilizando este y otros instrumentos, para conocer los motivos por las que se participa en este tipo de pruebas, existe una amplia literatura en población latina (López de la Llave, Pérez-Llantada, \& Buceta, 2002; Llopis \& Llopis, 2006; 2008; Jaenes, 1994; Jaenes \& Caracuel, 
2005; Ruiz-Juan \& Zarauz, 2011a; Ruiz-Juan \& Zarauz, 2012a; Zarauz \& Ruiz-Juan, 2013a; Zarauz \& Ruiz-Juan, 2014a) como anglosajona (Deaner, Masters, Ogles, \& LaCaille, 2011; Doppelmayr \& Molkenthin, 2004; Hall, Kerr, Kozub, \& Finnie, 2007; Thornton \& Scott, 1995).

Carmack y Martens (1979) clasificaron las razones para comenzar y mantenerse en carrera continua (jogging) en ocho categorías: salud física, salud psicológica, logro de metas, recompensas tangibles, influencias sociales, disponibilidad y motivos diversos. Por su parte Clough, Shepherd y Maughan (1989) analizaron los motivos de práctica recreativa del maratón y los agruparon en seis categorías: bienestar, sociales, desafío, estatus físico/salud y adicción. Ahora bien, ha de observarse que tanto los jóvenes como los adultos participan en el deporte por muy diversos motivos (Campbell, MacAulay, McCrum \& Evans, 2001; Weiss \& Ferrer-Caja, 2002; Williams, 2013). Por su parte Doppelmayr \& Molkenthin (2004), observaron que los corredores de ultramaratón de aventura se diferenciaban de los corredores de maratón, en una mayor presencia de los motivos naturales (disfrute del medio natural, etc.), disfrute de la sensación de vivir en un entorno de reto, y un menor interés por la obtención de marcas o de reto competitivo.

Un constructo que tomó importancia en el mundo del deporte y significativamente en la especialidad de carreras de larga distancia, es el compromiso a correr (CC). Dicho concepto fue equiparado por Carmack y Martens (1979) con el de la adicción positiva a correr (APC). Esta vino a definirse como una actividad agradable causante de placer extremo, incluso euforia, con unos efectos mentales que hacen que la experiencia sea tan placentera como adictiva (Glasser, 1976); frente a una adicción negativa a correr (ANC), que presentaría sus efectos más indeseables. Si bien, Horton \& Mack (2000) consideraban que no tiene por qué existir una asociación entre el CC y la ANC, otros sin embargo entendieron que la ANC solía ser una consecuencia del CC (Dawson \& Peco, 2004; Leedy, 2000).

Recientemente, el trabajo de Zarauz y Ruiz-Juan (2012) ha mostrado que, tanto en hombres como en mujeres, las motivaciones facilitadoras para el CC, clasificados según las MOMS-34, eran del tipo el significado de la vida-autoestima. Dicha escala era la que alcanzaba puntuaciones superiores a las reflejadas por la superación de metas personales-competición, al igual que lo mostrado anteriormente por Ogles y Masters (2003). Junto al significado de la vida-autoestima, se mostró que las metas psicológicas y los motivos relacionados con la salud eran predictores de un alto compromiso positivo con el correr en los hombres, siendo la segunda variable más valorada entre las mujeres. Sin embargo, la motivación relacionada con la superación de metas personales-competición, aunque muy valorada, fue la variable que predeciría la 'súper-adherencia', la adicción negativa a correr en los hombres del estudio de Zarauz y Ruiz-Juan (2012).

Ruiz-Juan y Zarauz (2012b) y Zarauz y RuizJuan (2011b) obtuvieron que las dos variables que mejor predecían un mayor CC son el número de kilómetros y los días entrenados a la semana, coincidiendo con lo reflejado por Thornton y Scott (1995). Estos últimos consideraron que las esferas primarias de motivación en el corredor comprometido (committed runner) se centraban en el dominio (mastery) y la competición. Establecieron que los motivos de práctica más destacados fueron: el dominio, la competición, la regulación del peso, la preocupación por la salud y el acondicionamiento físico. Siendo el dominio y el reconocimiento social buenos predictores de las altas puntuaciones en adicción.

Un aspecto especialmente importante en la práctica deportiva es la perspectiva de meta que una persona adopta en un ambiente concreto y esta dependerá de una serie de factores situacionales y de diferencias personales en la propensión a los diferentes tipos de implicación (Duda, 1995; Biddle, 1997; Escartí \& Cervelló, 1994). Así, se observa una correlación positiva entre la orientación a la tarea y la creencia de éxito deportivo en función de factores motivacionales y de trabajo duro (motivaciónesfuerzo) y negativa con la visión de que la habilidad para hacer trampa y engañar al entrenador lleva al triunfo deportivo. Mientras la orientación al ego correlaciona positivamente con la creencia de que 
ser un deportista más hábil y tener más talento llevaba al éxito (Salinero, Ruiz, \& Sánchez-Bañuelos, 2006), si bien también muestra relaciones con el desarrollo de procesos de dependencia al ejercicio (González \& Sicilia, 2012; Zarauz, Ruiz-Juan, \& Arbinaga, 2014).

Por otro lado, han cobrado especial relevancia las relaciones entre la ansiedad y la motivación para la práctica de ejercicio o el deporte; haciendo especial hincapié en su doble variante cognitiva y somática (Davidson, Schwartz, \& Goleman, 1978). Se ha mostrado, por un lado, que los deportistas de equipo indicaban menores niveles de ansiedad que los deportistas individuales (Furst \& Tenembaum, 1984; Ruiz-Juan \& Zarauz, 2014) y, por otro, que las relaciones que mostraban los niveles de ansiedad con la percepción que tenía el deportista del éxito, venían a señalar que una mayor ansiedad se observaba en deportistas con una alta orientación al ego y poca autoconfianza (Voigh, Callaghan, \& Ryska, 2000). Por su parte, Cervelló, Santos-Rosa, Jiménez, Nerea y García (2002) matizaron que la orientación disposicional al ego predice de manera negativa la ansiedad cognitiva, mientras que la orientación a la tarea predice significativa y positivamente la autoconfianza (Ruiz-Juan \& Zarauz, 2013b; Zarauz \& Ruiz-Juan, 2014b).

En todo este contexto, se busca determinar qué variables permitirán diseñar un modelo predictivo de los factores motivacionales alegados para la práctica de la carrera de larga distancia de manera diferencial según el contexto cultural y el sexo del corredor. Teniendo presente, por la importancia que ha venido mostrando en la literatura, la orientación motivacional y los resultados obtenidos en la evaluación de la ansiedad competitiva y el compromiso a correr. De esta forma y considerando las sugerencias de Deaner, Masters, Ogles y LaCaille (2011) al documentar diferencias debidas al sexo y mediadas por el tipo de deporte, los períodos temporales y la cultura, se abren nuevas perspectivas en la expresión de las diversas vertientes motivacionales. Por ello, se busca determinar la posible existencia de influencias culturales diferenciadoras en la contextualización de dichos modelos entre corredores españoles y mexicanos.

\section{Método}

\section{Participantes}

Se partió del total de corredores inscritos en los medios maratones de Almería y Elche (España) y Guadalajara (México) en 2010. Para asegurar que la muestra fuera representativa del total de estas carreras (error $\pm 3 \%$, intervalo de confianza 95.5\%), se empleó un diseño de muestreo estratificado por afijación proporcional teniendo en cuenta el sexo ( $86.65 \%$ de hombres y $13.35 \%$ de mujeres) y la edad. Se administró un cuestionario a 1.054 corredores de fondo en ruta que participaron en los medios maratones de Almería (30/01/2011), Elche $(03 / 04 / 2011)$ y dos ediciones de Guadalajara (20/02/2011; 19/02/2012).

Además por medio de una página Web se obtuvieron respuestas voluntarias de 741 cuestionarios de corredores de maratón y medio maratón desde 01/03/11 a 20/03/13. Por tanto, uniendo la muestra aleatoria y la voluntaria, se obtuvo una muestra total de 1.795 (1.105 españoles, 690 mexicanos) corredores de fondo en ruta, que estaba compuesta por 1.541 hombres $(85.65 \%)$ con rango de edad de 18 a 76 años $(M=38.98$; $D T=10.45)$, y 254 mujeres $(14.35 \%)$ con rango de edad de 18 a 69 años $(\mathrm{M}=37.88 ; \mathrm{DT}=9.80)$.

\section{Procedimiento}

En las carreras citadas anteriormente, se pidió permiso a la organización de la carrera mediante una carta en la que se explicaban los objetivos de la investigación, cómo se iba a realizar el estudio y se acompañaba un modelo del instrumento. El cuestionario fue administrado en un stand que se dispuso al efecto durante la recogida de dorsales de los atletas participantes el día previo a la carrera.

Para ampliar la muestra y obtener la más amplia y variada geográficamente en el territorio español, se solicitó colaboración al webmaster del principal foro de atletismo de España (El Atleta, s.f.), al que se le pidió que publicase el cuestionario en la sección de corredores de ruta ((Retos Nuevas Tendencias en Educación Física Deportes y Recreación, s.f.). 
En ambos casos, todos los sujetos fueron informados del objetivo del estudio, de la voluntariedad, absoluta confidencialidad de las respuestas y manejo de los datos, que no había respuestas correctas o incorrectas y se les solicitó que contestaran con la máxima sinceridad y honestidad. Además, se pidió que si habían contestado previamente este cuestionario no lo volvieran a hacer. Este trabajo posee el informe favorable de la Comisión de Bioética de la Universidad de Murcia.

\section{Instrumentos}

- Motivations of Marathoners Scales-34 (MOMS-34) de Ruiz-Juan y Zarauz (2011a), versión española de las Motivations of Marathoners Scales (MOMS) de Masters et al. (1993). Contiene siete subescalas con 34 ítems que se interesan por los motivos para correr: Orientación a la salud, peso, superación de metas personales-competición, reconocimiento, afiliación, metas psicológicas y significado de la vida-autoestima. Las respuestas se recogen en una escala tipo Likert desde 1 (no es una razón para correr) hasta 7 (es una razón muy importante para correr), con puntuación total media en cada escala entre 1 (mínima motivación para correr) y 7 (máxima motivación para correr).

- Commitment to Running Scale-11 (CR-11) de Ruiz-Juan y Zarauz (2011b), versión española de la Commitment to Running Scale (CR) de Carmack y Martens (1979). Contiene once ítems para medir el compromiso a correr (CC) puntuables de 1 (nada de acuerdo) hasta 5 (totalmente de acuerdo), con el que se obtiene una puntuación total entre 11 (mínimo CC) y 55 (máximo CC).

- Inventario de Ansiedad Competitiva-2 Revisado, versión española de Andrade, Lois y Arce (2007) de Revised Competitive State Anxiety Inventory-2 (CSAI-2R) de Cox et al. (2003). Tiene tres subescalas: ansiedad cognitiva, ansiedad somática y autoconfianza. La primera y tercera contienen cinco ítems puntuables de 1 (nada) a 4 (mucho) con la que se obtiene una puntuación total entre 5 y 20. La segunda contiene seis ítems y ofrece puntuaciones entre 6 y 24 .
- Cuestionario de Percepción de Éxito, versión española de Cervelló (1996) del Perception of Success Questionnaire (POSQ) de Roberts y Balagué (1991). Fue elaborado para medir la orientación de las metas de logro en el contexto deportivo. Consta de doce ítems, seis de orientación en la tarea y otras seis en el ego. La pregunta inicial que encabeza el cuestionario es "Siento éxito en el deporte cuando...". Las respuestas se recogen en escala tipo Likert que oscilan desde totalmente en desacuerdo (1) a totalmente de acuerdo (5).

\section{Análisis de los datos}

Correlación entre las subescalas (coeficiente de Pearson), consistencia interna (alfa de Cronbach), diferencias de medias por países y sexo ( $t$ Student) y regresión lineal multivariante, se realizaron con SPSS 20.0.

\section{Resultados}

\section{Estadística descriptiva}

Con respecto a las MOMS-34, conviene reseñar que la muestra mexicana de corredores de fondo en ruta presentó, en todas las subescalas y de manera significativa, valores medios superiores a la muestra española. Dichas diferencias también se apreciaron tanto en hombres como en mujeres (Tabla 1). Las diferencias entre sexos en un mismo país, vienen a indicar que la población española es diferente en dos de las subescalas de las MOMS-34, en las cuales los hombres obtuvieron mayores puntuaciones, siendo estas el peso $(t=3.34, p=0.001)$ y las metas personales-competición $(t=2.18, p=0.029)$. Por su parte, en la población mexicana, fue en la escala de metas personales-competición donde los hombres puntuaron por encima de las mujeres $(t=3.40$, $p=0.001)$.

La puntuación total de la CR-11 fue sensiblemente superior a la media de la escala, siendo considerablemente más elevada en la muestra mexicana $(t=22.91, p<0.000)$ (Tabla 1). Al considerarse por países y sexo, se observó que tanto las mujeres españolas $(t=2.67, p=0.008)$ como las mexicanas 
$(t=2.66, p=.008)$ obtuvieron puntuaciones superiores a las de los hombres de sus respectivos países.

Tanto los corredores españoles como mexicanos presentaban valores por debajo de la media en ansiedad cognitiva y ansiedad somática, y por encima en autoconfianza. Al considerarse entre ambos países, fue posible observar que solo se presentaron diferencias en las subescalas de ansiedad somática $(t=2.03$, $p=0.042)$ y autoconfianza $(t=14.28, p<0.000)$, donde fueron los españoles en la primera y los mexicanos en la segunda subescala quienes puntuaron por encima (Tabla 1). Teniendo presente el sexo por países, solo se concretaron diferencias en $a u$ toconfianza, donde los hombres españoles $(t=2.20$, $p=0.028)$ y los mexicanos $(t=2.06, p=0.039)$ obtuvieron mayores puntuaciones que las mujeres.

Por último, en cuanto a la percepción de éxito en el deporte, ambas orientaciones de meta estuvieron próximas a la media, si bien los corredores mexicanos presentaron medias superiores y estadísticamente significativas a los españoles, tanto en ego $(t=10.89, p<0.000)$ como en tarea $(t=10.64$, $p<0.000$ ). Según el sexo en cada país, no se apreciaron diferencias estadísticamente significativas entre hombres y mujeres (Tabla 1).

\section{Relaciones de los motivos para correr con compromiso, ansiedad precompetitiva y percepción de éxito en el deporte}

La orientación a la salud correlacionó positiva y significativamente, en los hombres de ambos países, con CC, ansiedad cognitiva, ansiedad somática, autoconfianza, ego y tarea (Tabla 2). En las mujeres de ambos países, correlacionó con CC, autoconfianza, ego y tarea (Tabla 3).

El peso en los hombres de ambos países, correlacionó positiva y significativamente con $\mathrm{CC}$, ansiedad cognitiva, ansiedad somática, ego y tarea (Tabla 2). En las mujeres se observaron diferencias, ya que en España no correlacionó con ninguna variable, mientras en México lo hizo solo con ego y tarea (Tabla 2).

Las metas personales-competición correlacionaron positiva y significativamente con todas las subescalas en los hombres de ambos países (Tabla
2). Por el contrario, en las mujeres españolas y mexicanas, las correlaciones significativas se dieron con CC, ansiedad cognitiva, autoconfianza (solo en México), ego y tarea (Tabla 3).

Tanto en hombres españoles como mexicanos, el reconocimiento correlacionó positiva y significativamente con CC, ansiedad cognitiva, ansiedad somática, ego y tarea (Tabla 2). Sin embargo, para las mujeres españolas y mexicanas la correlación fue con ansiedad cognitiva, ego y tarea (Tabla 3).

La afiliación correlacionó positiva y significativamente con todas las subescalas en los hombres de ambos países (Tabla 2). Por el contrario en las mujeres de ambos países las correlaciones significativas se dieron con autoconfianza, ego y tarea (Tabla 3).

Tanto en españoles como en mexicanos, las metas psicológicas correlacionaron positiva y significativamente con todas las subescalas (Tabla 2). En las mujeres se observaron bastantes diferencias ya que en ambos países, solo correlacionó con ego y tarea (Tabla 3).

Por último, indicar que, en los hombres de ambos países, se encontró correlación positiva y significativa de significado de la vida-autoestima con todas las subescalas (Tabla 2). Sin embargo en las mujeres de ambos países solo correlacionó con CC, autoconfianza, ego y tarea (Tabla 3).

\section{Análisis regresivo multivariante}

Se realizó un análisis de regresión lineal multivariado, tratando de obtener unos modelos que explicasen la mayor parte posible de varianza. Se tomaron como variables dependientes las puntuaciones medias de las siete subescalas de las MOMS-34. Las variables predictoras fueron el CC, cada una de las subescalas de ansiedad precompetitiva (ansiedad cognitiva, ansiedad somática y autoconfianza) y de la percepción de éxito en el deporte (ego y tarea). Como variable de selección se consideró el sexo y el país.

Se extrajo el valor $R^{2}$ para explicar la varianza, Beta para explicar la predicción entre variables, $F$ para ver si existe relación entre variables seleccionadas y su significatividad (Tabla 4 y 5). Se obtuvieron sólidos modelos, ya que explicaron entre el cuarenta 


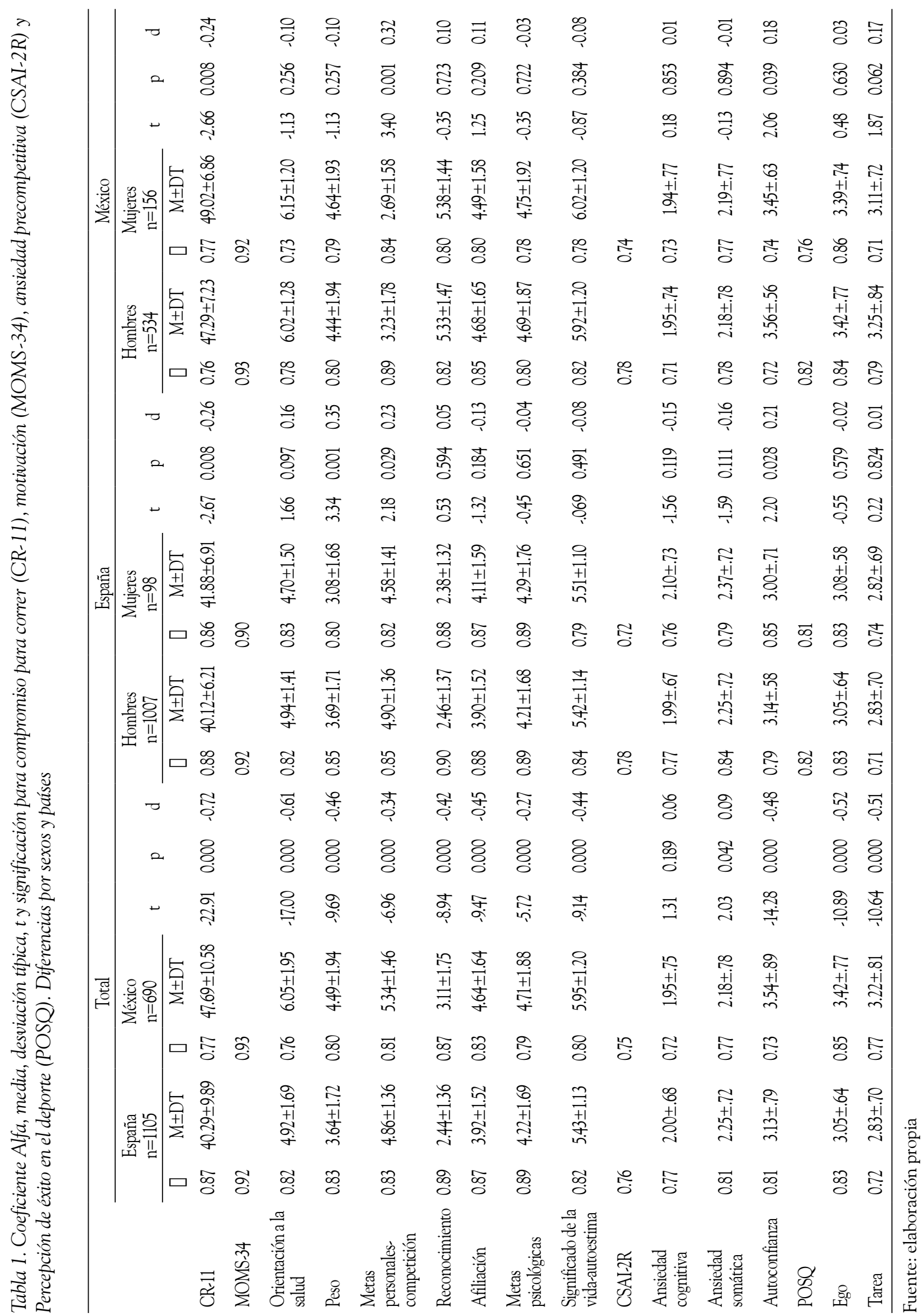


Antonio Zarauz Sancho, Francisco Ruiz-Juan, Félix Arbinaga Ibarzábal, José Carlos Jaenes SÁnchez, Gabriel Flores-Allende Correo
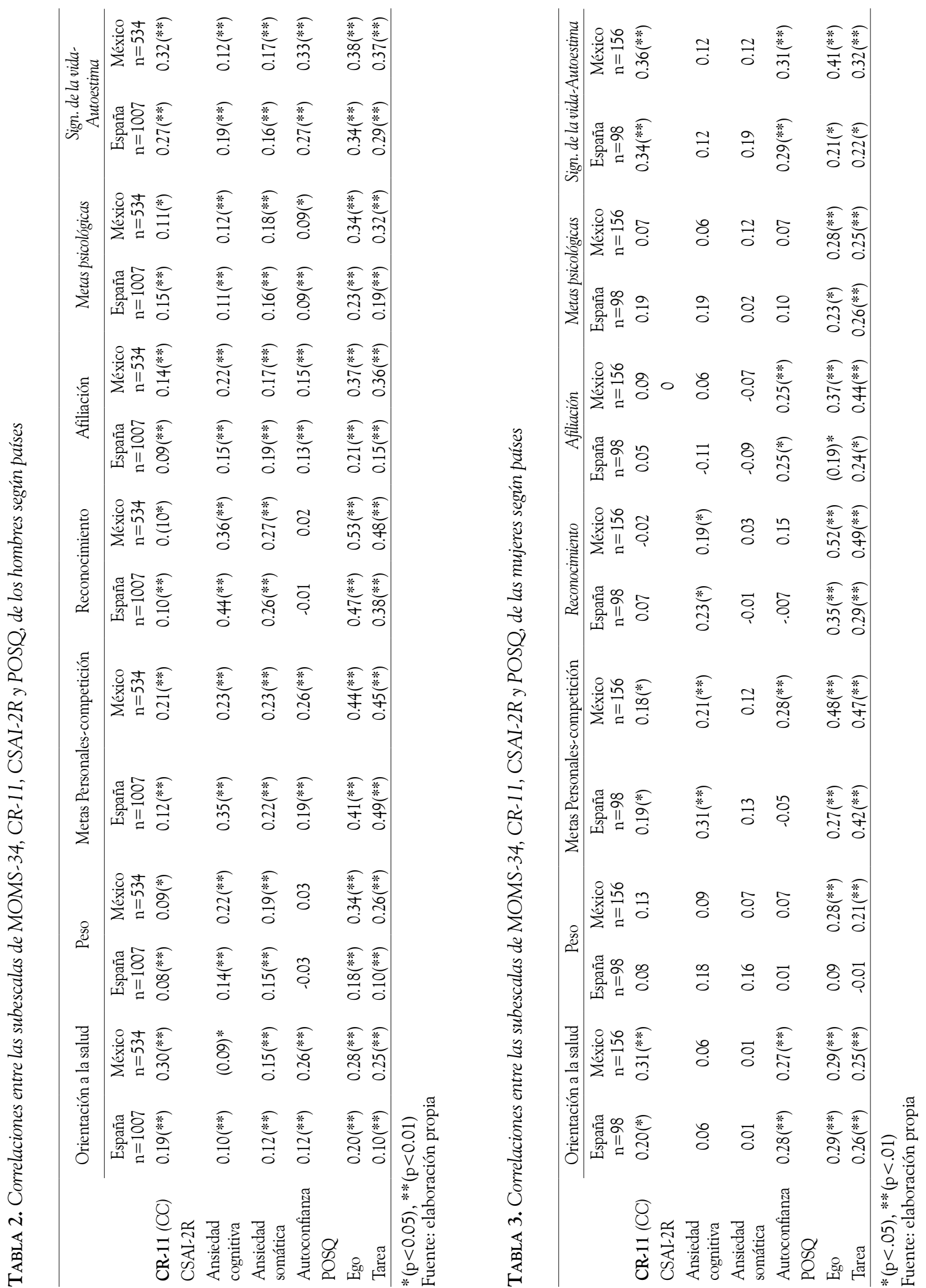
y el sesenta por ciento de la varianza en hombres y mujeres de ambos países.

El modelo de Orientación a la salud en hombres de ambos países, fue idéntico. Se pudo predecir significativamente por puntuar alto en CC, ansiedad somática, autoconfianza y ego (varianza: 40.5\% España, 42.3\% México). También fue totalmente similar el modelo en las mujeres de ambos países, pero con diferencias con los hombres. La predicción fue por puntuar alto en CC y autoconfianza (varianza: 44.8\% España, 43.6\% México).

En los hombres de ambos países, el modelo del peso fue muy parecido. Se pudo predecir por puntuar alto en CC (solo en España), ansiedad somática y ego (varianza: 46.2\% España, 47.3\% México). En las mujeres de ambos países, el modelo fue el mismo, es decir, se podía predecir por puntuar bajo en CC (varianza: 50.6\% España, 42.8\% México).

El modelo de metas personales-competición en hombres de ambos países fue también el mismo. Se podía predecir significativamente por puntuar alto en CC, ansiedad cognitiva, autoconfianza, ego y tarea (varianza: 55.9\% España, 54.8\% México). También fue casi similar el modelo en las mujeres de ambos países pero con diferencias con los hombres. La predicción fue por puntuar alto en ansiedad cognitiva, autoconfianza (solo en México) y tarea (varianza: 50.4\% España, 59.3\% México).

En los hombres de España y México, el modelo del reconocimiento fue bastante parecido. Se podía predecir por puntuar alto en CC, ansiedad cognitiva, ego y tarea (solo en México) (varianza: 54.8\% España, 59.5\% México). En las mujeres de ambos países, el modelo fue casi el mismo, es decir, se podía predecir por puntuar alto ego y tarea (solo en México) (varianza: 43.7\% España, 60.1\% México).

El modelo de Afiliación, en hombres de ambos países, fue también prácticamente el mismo. Se podía predecir significativamente por puntuar alto en CC, ansiedad somática, autoconfianza, ego y tarea (solo en México) (varianza: 49.8\% España, 43.5\% México). El modelo en las mujeres de ambos países fue similar pero con diferencias importantes con los hombres. La predicción fue por puntuar alto solo en tarea (varianza: 46.8\% España, 50.5\% México).
En los hombres de ambos países, el modelo de metas psicológicas fue casi idéntico. Se podía predecir por puntuar alto en CC, ansiedad somática, ego y tarea (solo en México) (varianza: 49.6\% España, 47.1\% México). En las mujeres de ambos países el modelo fue el mismo, pero diferente a los hombres, ya que no fue posible predecirlo por ninguna variable (varianza: 47.8\% España, 43.6\% México).

Por último, el modelo de Significado de la vidaAutoestima fue idéntico en los hombres de ambos países. Se podía predecir por puntuar alto en CC, ansiedad somática, autoconfianza y ego (varianza: 50.8\% España, 53.5\% México). En las mujeres de ambos países, el modelo fue el mismo, pero diferente a los hombres, es decir, se podía predecir por puntuar alto en CC, ansiedad somática y autoconfianza (varianza: 54.1\% España, 55.1\% México).

\section{Discusión}

Manteniendo presente la sugerencia de Deaner, Masters, Ogles y LaCaille (2011), de que se enriquece el estudio y análisis de los motivos y los procesos motivacionales al considerar las diferencias debidas al sexo, el tipo de deporte y la cultura, se ha buscado determinar de manera predictiva, los motivos de práctica de carrera en ruta, analizando la posible existencia de influencias culturales diferenciadoras en la contextualización de dichos modelos entre corredores españoles y mexicanos, mediados por el sexo de los mismos.

A partir de los datos aportados, es posible comprobar que el contexto cultural marca diferencias importantes entre las dos nacionalidades. El grupo formado por los participantes con nacionalidad mexicana nos dejó constancia de mayores puntuaciones en todas las subescalas de las MOMS-34, en la CR-11, en el POSQ y en una de las tres subescalas del CSAI-2R, en otra puntuaría menos y en la tercera no se indicarían diferencias.

Es importante señalar que la valoración realizada para la adicción positiva a correr, obtenida mediante el pase de la prueba CR-11, viene a indicar que la población mexicana mostró mayores puntuaciones siendo, tanto los hombres como las mujeres, quienes así lo indicaron. Al considerar el 
Antonio Zarauz Sancho, Francisco Ruiz-Juan, Félix Arbinaga Ibarzábal, José Carlos Jaenes Sánchez, Gabriel Flores-Allende Correo
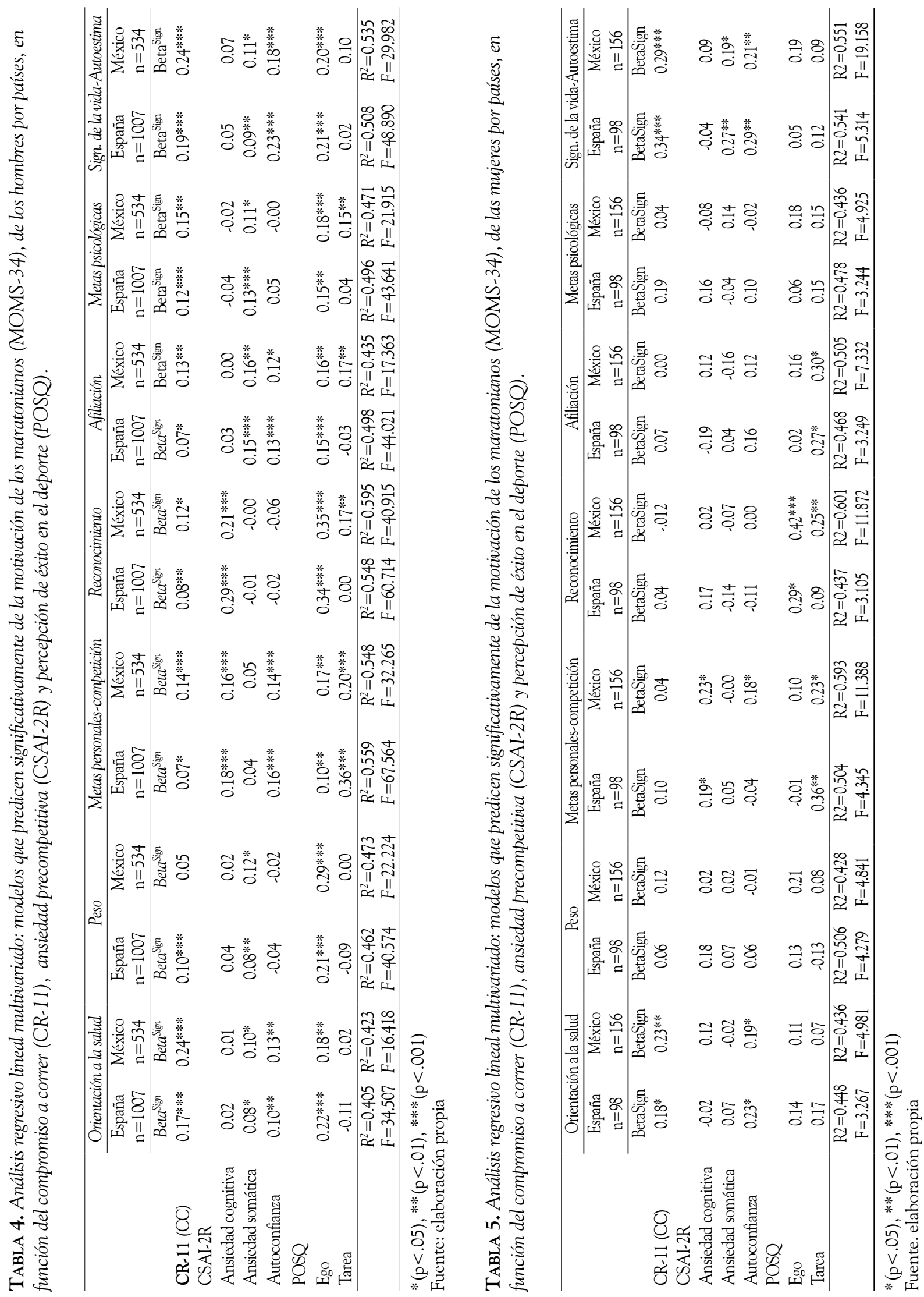
sexo dentro de cada país, los datos avalaron lo que ya se venía mostrando en trabajos anteriores (RuizJuan \& Zarauz, 2011b; Zarauz \& Ruiz-Juan, 2011a; Zarauz \& Ruiz-Juan, 2012; Zarauz \& Ruiz-Juan, 2013c) al encontrar que las mujeres muestran un mayor compromiso a correr.

De otra parte, mediante las puntuaciones obtenidas en el CSAI-2R, se observó que las poblaciones de ambos países no se diferenciaban en ansiedad cognitiva, pero sí en ansiedad somática y autoconfianza. En el caso de esta última, fue la población mexicana la que mostró una mayor autoconfianza pero no una mayor ansiedad somática. Este hecho, según Larumbe, Pérez-Llantada y López de la Llave (2009), puede quedar explicado porque los corredores con mayor autoconfianza son aquellos que perciben que están mejor entrenados para afrontar la carrera (Ruiz-Juan \& Zarauz, 2013a; 2013b), cosa que se desprende de los datos del compromiso significativamente mayor obtenido por los mexicanos, todo lo cual les genera igualmente una motivación significativamente mayor para competir respecto a los españoles.

Mediante las puntuaciones obtenidas en la orientación disposicional - ego/tarea- la población mexicana ha mostrado una puntuación superior a la reflejada por la muestra española en ambas orientaciones dentro del contexto deportivo; no detectándose diferencias entre hombres y mujeres. Resultados parcialmente similares a los aportados por Moreno, Cervelló y González (2007), Hall, Kerr, Kozub y Finnie (2007), Elliot y McGregor (2001). Estos últimos partiendo de la idea sugerida de que la ANC puede ser una consecuencia del CC (Dawson \& Peco, 2004; Leedy, 2000), observaron que el perfil motivacional de los atletas que se asociaba con la ANC era una alta orientación al ego combinada con una moderada orientación a la tarea; presentando aspectos diferenciales entre los hombres y las mujeres. Los datos constatados en este trabajo, avalan dichas consideraciones al mostrar un peso diferencial entre la orientación al ego y la orientación a la tarea como variable predictora de los motivos alegados para correr y en función del sexo de los participantes. Así, para los hombres se ha observado una alta significación del aporte de la orientación al ego como variable predictora en todos los motivos recogidos en las MOMS-34; mientras la orientación a la tarea se mostraría con una aportación significativa para los motivos de competición, reconocimiento, afiliación y metas psicológicas. Mientras en el caso de las mujeres la orientación al ego realizó una aportación significativa solo a los motivos de reconocimiento; la orientación a la tarea lo hizo a los motivos de metas personales-competición, afiliación y reconocimiento (este último solo en las mujeres mexicanas).

Por último, teniendo en cuenta el sexo de los participantes en la muestra, se observaron diferencias entre los hombres y mujeres mediadas por el factor país en sus respuestas a las MOMS-34. Así, los españoles obtuvieron mayores puntuaciones frente a las mujeres de la misma nacionalidad, en la variable peso y en la variable metas personales-competición; mientras en la población mexicana solo se detectaron diferencias en las puntuaciones obtenidas en la variable metas personales-competición, donde fueron los hombres mexicanos quienes obtuvieron mayores puntuaciones. Estos datos apoyan parcialmente lo mostrado por Ogles, Masters y Richardson (1995) quienes encontraron que los hombres señalaban más que las mujeres la dimensión de motivaciones de logro (competición y logro de metas personales) y la subescala de reconocimiento. Ahora bien, fueron ellas quienes obtenían mayores puntuaciones en peso, afiliación, autoestima, metas psicológicas y sentido de la vida. Sin embargo, los datos recogidos no apoyaron las mismas diferencias que las indicadas por Ruiz-Juan y Zarauz (2011a) quienes dejaban constancia de diferencias solo en reconocimiento y significado de la vida-autoestima donde, respectivamente, hombres y mujeres mostraron mayores puntuaciones.

Si bien se detectaron diferencias entre los corredores de ambos países, donde los mexicanos obtuvieron mayores valores en prácticamente casi todas las escalas y subescalas, ha de decirse que los modelos predictivos basados en los análisis de regresión fueron muy similares, cuando se comparan las dos nacionalidades agrupadas por el sexo del participante. Puede afirmarse que, tanto para los hombres españoles vs. mexicanos, como para 
las mujeres españolas vs. mexicanas, los modelos que predicen cada una de las subescalas de las MOMS-34 están conformados por casi las mismas subescalas dentro de cada sexo y entre países; pero muy diferentes cuando se comparan entre sexos. En este sentido, se ha mostrado que los modelos, en hombres españoles y mexicanos, que predicen las motivaciones de Significado de la vida-autoestima, orientación a la salud y metas personales-competición, son exactamente iguales. Sin embargo, mostraron pequeñas diferencias en peso, reconocimiento, afiliación y metas psicológicas.

En el caso de las mujeres, si bien fueron diferentes todos los modelos a los mostrados por los hombres, se observó que las diferencias entre españolas y mexicanas se presentaban en los modelos que predecían las motivaciones relacionadas con las metas personales-competición y reconocimiento. Por su parte, fueron iguales los modelos predictivos de las motivaciones de afiliación, metas psicológicas, significado de la vida-autoestima, orientación a la salud y peso. Por tanto, se estaría hablando de diferencias entre sexos y de intensidad entre los países, pero se dispondría de similitudes en cuanto a las variables o dimensiones implicadas. Todo ello sugiere que la motivación a la hora de realizar un tipo de ejercicio es multifacética y se refieren a beneficios claves que las personas quieren alcanzar a través del ejercicio (Mullen \& Markland, 1997).

Estas diferencias que se observaron entre los hombres y las mujeres, independientemente del país o contexto cultural donde se produjeron, vendrían a apoyar las afirmaciones realizadas por Deaner, Masterds, Ogles y LaCaille (2011) en cuanto a las diferencias en el rendimiento relativo que ellos observaron entre los hombres y las mujeres, tanto en la competición como en el compromiso con los entrenamientos. Estos hallazgos parecen mostrar que, aunque podría haber una diferencia de género intrínseca en la competitividad de los deportes, la intensidad de sus diferencias dependería de factores socioculturales. Ahora bien, sí sería de interés analizar las circunstancias contextuales que definen tales diferencias de intensidad en las puntuaciones obtenidas entre los corredores de ambos países.
Por lo tanto, los estudios futuros han de documentar cómo las diferencias de género en el desempeño relativo en una actividad deportiva se ven mediadas por factores culturales. Por ello, han de proporcionar un mayor desarrollo y profundización en la expresión del compromiso de formación y competitividad deportiva.

\section{Referencias}

Allied Dunbar National Fitness Survey (1992). Allied Dunbar National Fitness Survey main findings. London: Sports Council and Health Education Authority.

Andrade, E. M., Lois, G., \& Arce, C. (2007). Propiedades psicométricas de la versión española del Inventario de Ansiedad Competitiva CSAI-2R en deportistas. Psicothema, 19(1), 150-155.

Biddle, S. (1997). Cognitive theories of motivation and the physical self. En K. Fox (ed.). The physical self (pp: 59-82). Champaign: Human Kinetics.

Campbell, P. G., Macaulay, D., McCrum, E., \& Evans, A. (2001). Age differences in the motivating factors for exercise. Journal of Sport and Exercise Psychology, 23, 3, 191-199

Carmack, M. A., \& Martens, R. (1979). Measuring commitment to running: A surrey of runner's attitudes and mental status. Journal of Sport and Exercise Psychology. 1, 25-42.

Castillo, L, Balaguer, I., \& Duda, J. (2000). Las orientaciones de meta y los motivos de práctica deportiva en los jóvenes deportistas valencianos escolarizados. Revista de Psicología del Deporte, 9(1-2), 37-50.

Cervelló, E. (1996). La motivación y el abandono deportivo desde la perspectiva de las metas de logro. Tesis Doctoral. Universitat de València, Valencia.

Cervelló, E. Santos-Rosa, F. J., Jiménez, R., Nerea, A., \& García, T. (2002). Motivación y ansiedad en jugadores de tenis. Motricidad, 9, 141-161.

Clough, P., Shepherd, J., \& Maughan, R. (1989). Motives for participation in recreational running. Journal of Leisure Research, 21, 297-309.

Cox, R., Martens, M., \& Russell W. (2003). Measuring anxiety in athletics: The revised Competitive State Anxiety Inventory-2. Journal of Sport and Exercise Psychology, 25, 519-533. 
Crandall, R. J. (1980). Motivations for leisure. Journal of Leisure Research, 12, 45-54.

Davidson, G. E., Schwartz, R. J., \& Goleman, D. J. (1978). Patterning of cognitive and somatic processes in self-regulation of anxiety: effects of meditation versus exercise. Psychosomatic Medicine, 40, 321-328.

Dawson, K. A., \& Peco, J. (2004). Exercise Motivation, Commitment, Addiction and Gender. Medicine $\mathcal{E}$ Science in Sports $\mathcal{E}$ Exercise, 36, 64-65.

Deaner, R., Masters, K., Ogles, B., \& LaCaille, R. (2011). Marathon performance as a predictor of competitiveness and training in men and women. Journal of Sport Behavior, 34(4), 325-342.

Doppelmayr, M., \& Molkenthin, A. (2004). Motivations of participants in adventure ultramarathons compared to other foot races. Biology of Sport, 21(4) 319-323.

Duda, J. (1995). Motivación en los escenarios deportivos: Un planteamiento de perspectiva de meta. En G. Roberts (Ed.). Motivación en el deporte y el ejercicio (pp. 85-122). Bilbao. DDB.

El Atleta (s.f.) Foro. Recuperado de: http://www.elatleta. com/foro/forum.php

Elliot, A. J., \& McGregor, H. A. (2001). A 2x2 achievement goal framework. Journal of Personality and Social Psychology, 80, 501-519.

Escartí, A., \& Brustad, R. (2000). El estudio de la motivación deportiva desde la perspectiva de la teoría de metas. I Congreso Hispano-Portugués de Psicología, Santiago de Compostela. España.

Escartí, A., \& Cervelló, E. (1994). La motivación en el deporte. En I. Balaguer (Ed.) Entrenamiento psicológico en el deporte (pp. 61-90). Valencia: Albatros educación.

Fisher, L. A. (2001). Case studies of female bodybuilders: culture gone awry? Proceedings of the Association for the Advancement of Applied Sport Psychology. 8-9 Denton, TX: Ronjon Publishing.

Furst, D. M., \& Tenembaum, G. (1984). Sport performance and various state anxiety components: A cross sectional study. Netanya: Wingate Institute.

Glasser, W. (1976). Positive Addiction. New York: Harper $\&$ Row.

González, D. \& Sicilia, A. (2012). Motivation and exercise dependence: a study base on self-determi- nation theory. Research Quarterly for Exercise and Sport, 83, 318-329.

González-Badillo, J. J., \& Goristiagoa, E. (2002). Fundamentos del entrenamiento de la fuerza: Aplicación al alto rendimiento deportivo. Barcelona: INDE.

González-Badillo, J. J., \& Ribas, J. (2002). Bases para la programación del entrenamiento de fuerza. Barcelona: INDE.

Gould, D. (1980). Motivating young athletes. Comunicación presentada en el Michigan Institute for the Study of Young Sports, Michigan.

Hall, H., Kerr, A., Kozub, S., \& Finnie, S. (2007). Motivational antecedents of obligatory exercise: The influence of achievement goals and multidimensional perfectionism. Psychology of Sport and Exercise, 8(3), 297-316. http://dx.doi.org/10.1016/j. psychspor.2006.04.007.

Horton, R. S., \& Mack, D. E. (2000). Athletic identity in marathon runners: functional focus or dysfunctional commitment? Journal of Sport Behavior, 23, 101-119.

Isorna Folgar, M., Rial Boubeta, A., \& Vaquero-Cristóbal, R. (2014). Motivaciones para la práctica deportiva en escolares federados y no federados. Retos, 25, 80-84.

Isorna Folgar, M., Rial Boubeta, A., Vaquero-Cristóbal, R., \& Sanmartín Zamácola, F. (2012). Motivaciones para la práctica de deporte federado y del piragüismo en alumnos de primaria y secundaria. Retos, 21, 19-24.

Jaenes, J. C. (1994). Psicología del deporte y maratón. En F. Plata, N. Terados \& P. Vera (Eds). El Maratón, aspectos técnicos y científicos (pp. 277-312). Madrid: Alianza Deporte.

Jaenes, J. C., \& Caracuel, J. C. (2005). Maratón: preparación psicológica para el entrenamiento y la competición. Córdoba: Almuzara.

Larumbe, E., Pérez-Llantada, C., \& López de la Llave, A. (2009). Características del estado psicológico de los corredores populares de maratón. Revista de Psicología del Deporte, 18(2), 151-163.

Leedy, M. G. (2000). Commitment to distance running: coping mechanism or addiction? Journal of Sport Behavior, 23, 255-270.

Llopis, D., \& Llopis, R. (2006). Razones para participar en carreras de resistencia. Un estudio con cor- 
redores aficionados. Cultura, Ciencia y Deporte, 2, 33-44.

Llopis, D., \& Llopis, R. (2008). Correr con cabeza. Claves psicológicas del maratón y las carreras de fondo. Alcoy: Altorendimiento.

López de la Llave, A., Pérez-Llantada, M. C., \& Buceta, J. M. (2002). Análisis de fiabilidad de las escalas del perfil psicológico óptimo para el deportista individual, versión para uso en Maratón (PODIVM): estudio preliminar. Metodología de las Ciencias del Comportamiento 2002. Volumen Especial. Madrid: AEMCCO.

López, C., \& Márquez, S. (2001). Motivación en jóvenes practicantes de lucha leonesa. Revista de Psicología del Deporte, 10, 9-22.

Martín-Albo, J., \& Núñez, J. L. (1999). La motivación deportiva: icuestión de tiempo? Revista de Psicología del Deporte, 8, 283-293.

Martín-Albo, L., Núñez. J. L., \& Navarro, J. G. (1997). Clasificación de los deportes en función de la evolución de los motivos atendiendo al tiempo de práctica y el género. Revista Electrónica de Motivación y Emoción, 5, 11-12.

Martín-Albo, J., Núñez, J. L., \& Navarro, J. G. (2003). La evolución motivacional como criterio discriminante en los deportes. Revista Latinoamericana de Psicología. 35(2), 139-150.

Masters, K. S., Ogles B. M., \& Jolton, J. A. (1993). The development of an instrument to measure Motivation for Marathon running: the Motivations of Marathoners Scales (MOMS). Research Quarterly for Exercise and Sport (RQES), 64, 134-143.

Moreno, J. A., Cervelló, E., \& González, D. (2007). Young athletes' motivational profiles. Journal of Sports Science and Medicine 6, 172-179

Mullen, E., \& Markland, D. (1997). Variations in selfdetermination across the stages of change for exercise in adults. Motivations and Emotion, 21, 349-362

Ogden, J., Veale, D., \& Summers, Z. (1997). The development and validation if the exercise dependence questionnaire. Addiction Research, 5, 343-356.

Ogles, B. M., \& Masters, K. S. (2003). A typology of marathon runners based on cluster analysis of motivations. Journal of Sport Behavior. 26(1),69-85

Ogles, B. M., Masters, K. S., \& Richardson, S. A. (1995). Obligatory running and gender: an analysis of par- ticipative motives and training habits. International Journal of Sports Psychology, 26, 233-248.

Retos Nuevas tendencias en Educación Física, Deportes y Recreación (s.f.) Cuestionario Aptitudes y Actitudes corredores de ruta. Recuperado de: http:// www.retos.org/encuesta/inicio.html

Roberts, G., \& Balagué, G. (1991, septiembre). The development and validation of the Perception of Success Questionnaire. Paper presented at the FEPSAC Congress, Cologne, Germany.

Ruiz-Juan, F., \& Zarauz Sancho, A. (2011a). Validación de la versión española de las Motivations of Marathoners Scales (MOMS). Revista Latinoamericana de Psicología, 43(1), 139-156.

Ruiz-Juan, F., \& Zarauz, A. (2011b). Validación de la versión española de la Commitment to Running Scale (CR). Estudios de Psicología, 32(2), 195-207.

Ruiz-Juan, F., \& Zarauz, A. (2012a). Predictor variables of motivation on Spanish master athletes. Journal of Human Sport and Exercise, 7(3), 617-628.

Ruiz-Juan, F., \& Zarauz, A. (2012b). Variables que hacen adicto negativamente a correr al maratoniano español. Retos, 21, 38-42.

Ruiz-Juan, F., \& Zarauz, A. (2013a). Análisis de la ansiedad en el atletismo; un estudio con veteranos. Revista Internacional de Ciencias del Deporte, 33(9), 222-235.

Ruiz-Juan, F., \& Zarauz, A. (2013b). Ansiedad, satisfacción, percepción y creencias sobre las causas del éxito en atletas veteranos españoles. Ansiedad y Estrés, 19(1), 83-93.

Ruiz-Juan, F., \& Zarauz, A. (2014). Ansiedad en maratonianos en función de variables socio-demográficas. Retos, 25, 28-31.

Sacks, M. H., \& Sachs, M. L. (1981). Psychology of running. Champaign: Morgantown.

Salinero, J. J., Ruiz, G., \& Sánchez-Bañuelos, F. (2006). Orientación y clima motivacional, motivación de logro, atribución de éxito y diversión en un deporte individual. Apunts Educación Física y Deportes, 83, 1, 5-11.

Thornton, E., \& Scott, S. (1995). Motivation in the committed runner: correlations between self-report scales and behaviour. Health Promotion International 10(3), 177-184. 
Vanek, M., \& Cratty, B. J. (1970). Psychology and the superior athlete. N.Y. Macmillan.

Voight, M. R., Callagham, J. L., \& Ryska, T. A. (2000). Relationship between goal orientations, self-confidence and multidimensional trait anxiety among Mexican-American female youth athletes. Journal of Sport Behavior, 23, 271-288.

Weiss, M. R., \& Ferrer-Caja, E. (2002). Motivational orientations and sport behaviour. En T. S. Horn (Ed.) Advances in sport psychology (pp. 101-183). Champaign: Human Kinetics.

Williams, L. (2013). Commitment to sport and exercise: re-examining the literature for a practical and parsimonious model. Journal of Preventive Medicine and Public Health, 46, S35-S42. http://dx.doi. org/10.3961/jpmph.2013.46.S.S35.

Zamarripa Rivera, J. I., Ruiz-Juan, F., López Walle, J. M., \& Fernández Baños, R. (2013). Actividad e inactividad física durante el tiempo libre en la población adulta de Monterrey (Nuevo León, México). Retos, 24, 91-96.

Zarauz, A., \& Ruiz-Juan, F. (2011a). Propiedades psicométricas de la versión española de la Running Addiction Scale (RAS). The Spanish Journal of Psychology, 14(2), 967-976.

Zarauz, A., \& Ruiz-Juan, F. (2011b). Compromiso y adicción negativa al entrenamiento y competición de los maratonianos. Revista Internacional de Medicina y Ciencias de la Actividad Física y el Deporte, 11(44), 817-834.

Zarauz, A., \& Ruiz-Juan, F. (2012). "Súper-adherencia” del maratoniano; variables predictoras y diferencias de género. Universitas Psychologica, 11(3), 863-874.

Zarauz, A., \& Ruiz-Juan, F. (2013a). Motivaciones de los maratonianos según variables socio-demográficas y de entrenamiento. Retos, 24, 50-56.

Zarauz, A., \& Ruiz-Juan, F. (2013b). Variables predictoras de la ansiedad en atletas veteranos españoles. Retos, 23, 29-32.

Zarauz, A., \& Ruiz-Juan, F. (2013c). Variables predictoras de la adicción al entrenamiento en atletas veteranos españoles. Retos, 24, 33-35.

Zarauz, A., \& Ruiz-Juan, F. (2014a). Análisis de la motivación en el atletismo; un estudio con veteranos. Universitas Psychologica, 13(2), 501-515.

Zarauz, A., \& Ruiz-Juan, F. (2014b). Factores determinantes de la ansiedad en atletas veteranos españoles. Universitas Psychologica, 13(3), 1047-1058.

Zarauz, A., Ruiz-Juan, F., \& Arbinaga, F. (2014). Adicción, satisfacción, percepción y creencias sobre las causas del éxito en atletas veteranos españoles. Adicciones, 26(2), 106-115. 
